\title{
Study of the tooth contact for high contact ratio spur gears with long tip relief
}

\author{
José I. Pedrero*, Miguel Pleguezuelos, and Miryam B. Sánchez \\ UNED, Departamento de Mecánica, Juan del Rosal 12, 28040 Madrid, Spain
}

\begin{abstract}
Profile modifications are commonly used to avoid shocks between meshing gear teeth produced by the delay of the driven gear, and the subsequent sooner start of contact, due to the teeth deflections. A suitable tip relief at the driven tooth shifts the start of contact to the proper location at the theoretical inner point of contact. The shape of the relief governs the loading curve of the tooth pair, while the length of relief determines the intervals in which this actual loading curve differs from the theoretical one of unmodified teeth. As at least one tooth pair should be in contact at the unmodified involute profile interval, the length of modification should be smaller than the length of the intervals of two pair tooth contact; otherwise, a shock at the end of contact of the previous pair is unavoidable. However this problem does not occur for high contact ratio spur gears, in which at least two couples of teeth are in contact at any moment. In this work, a study on the load sharing and the quasi-static transmission error for high contact ratio spur gears with long profile modification has been performed, and a model for the tooth contact has been developed.
\end{abstract}

\section{Introduction}

The tooth deflections under load induce a relative rotation between both meshing gears, delaying the driven gear respect to the driving one [1]. This delay produces a sooner start of contact of the subsequent couples of teeth, which occurs outside the pressure line, resulting in a shock between both meshing teeth which induces noise, vibrations and dynamic load. Previous studies on the load transfer along this extra contact interval reveal a parabolic loading process between the actual sooner start of contact and the theoretical inner point of contact [1, 2]. And a similar parabolic unloading process occurs at the upper limit of the contact interval $[2,3]$.

To avoid the shock at the beginning of the contact the profile modification is frequently used. In fact, a suitable tip relief at the driven tooth shifts the start of contact to the proper location [4]. Similarly, a symmetric tip relief at the driving tooth shifts the end of contact to the theoretical outer point of contact. However, this profile modification at the upper limit of the contact interval is not as critical as one at the inner limit because the parabolic unloading process induces a sudden disengage of meshing teeth but not a shock. It is therefore not unusual to modify the profile at the inner limit of the contact interval but not at the outer limit.

For such a profile modifications, the shape of the tip relief at the driven tooth governs the loading curve of the tooth pair [3-6], while the unloading curve is described by the mentioned parabola. However, for long profile modification, beyond the inner interval of minimum tooth pair contact, there is a contact interval in which both effects influence simultaneously, which obviously will have influence on the load sharing and the transmission error.

In this work, a study on the load sharing and the transmission error for spur gears with long profile modification has been performed. Though this kind of relieves is not suitable for standard contact ratio spur gears [3], a model for the tooth contact for high contact ratio gears has been developed.

\section{Unmodified teeth}

Theoretical contact between conjugate involute profiles provides uniform output velocities if input velocity is uniform too. However, real power transmissions with heavily loaded teeth lose the uniformity of the velocity ratio because of the teeth deflections -and subsequent delays in the output shafts- induced by the loads. As these delays are not equal along the meshing interval the output velocity becomes fluctuating and transmission error, or more properly the quasi-static transmission error (QSTE) arises unavoidably.

The load sharing, the tooth deflections and the QSTE are governed by the variation of the meshing stiffness along the path of contact. Accordingly, the analysis of the contact between unmodified involute teeth requires the following steps:

- Determination of the curve of meshing stiffness along the path of contact, from the hypothesis of ideal contact, inside the line of action (and valid only for unloaded or weakly loaded teeth).

- Determination of the curve of load sharing ratio (LSR) along the path of contact, from the obtained meshing stiffness. 


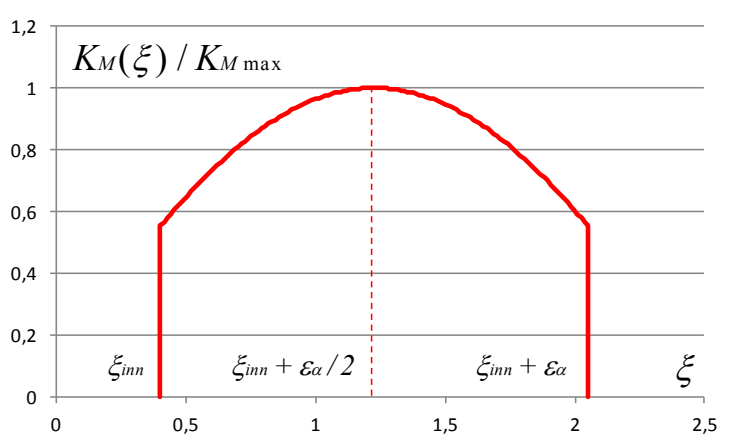

Fig. 1. Meshing stiffness $\left(\varepsilon_{\alpha}=1.65\right)$.

- Determination of the teeth deflections, according to the load at each point of the line of action, and the corresponding delay angle and QSTE.

- Determination of the effective interval of contact according to the delay angles at both limits of the interval.

- Determination of the LSR along the complete contact interval.

The curve of meshing stiffness under ideal contact conditions is very well known. Its shape is always as one shown in Fig. 1, and it is accurately described by [7]:

$$
K_{M}(\xi)=K_{M \max } \cos \left(b_{0}\left(\xi-\xi_{m}\right)\right)
$$

with:

$$
\begin{gathered}
b_{0}=\left[\frac{1}{2}\left(1.11+\frac{\varepsilon_{\alpha}}{2}\right)^{2}-1.17\right]^{-1 / 2} \\
\xi_{m}=\xi_{i n n}+\frac{\varepsilon_{\alpha}}{2}
\end{gathered}
$$

where $\varepsilon_{\alpha}$ is the contact ratio and $\xi_{i n n}$ and $\xi_{m}$ the pinion profile parameter $\xi$ corresponding to the inner point and the midpoint of the interval of contact, respectively, being $\xi$ :

$$
\xi=\frac{z_{1}}{2 \pi} \sqrt{\frac{r_{c 1}^{2}}{r_{b 1}^{2}}-1}
$$

in which $z$ is the number of teeth, $r_{c}$ the radius of the contact point, $r_{b}$ the base radius and subscript 1 denotes the driving gear.

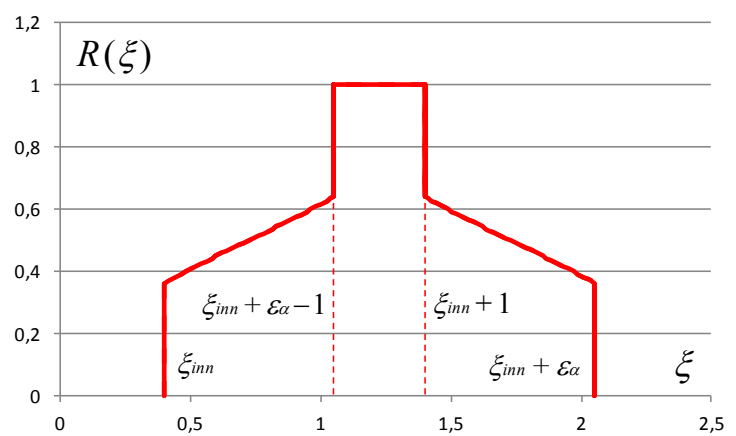

(a) Standard contact ratio $\left(\varepsilon_{\alpha}=1.65\right)$.
The load sharing ratio can be computed from $[3,7]$ :

$$
R(\xi)=\frac{F(\xi)}{F_{T}}=\frac{K_{M}(\xi)}{\sum_{j} K_{M}(\xi+j)}
$$

where the sum is extended to all the teeth in simultaneous contact. If $E_{\alpha}$ denotes the integer part of $\varepsilon_{\alpha}$ and considered $R(\xi)=0$ for values of $\xi$ outside the contact interval (which is described by $\xi_{\text {inn }} \leq \xi \leq \xi_{o}=\xi_{\text {inn }}$ $\left.+\varepsilon_{\alpha}\right), j$ in eqn. (4) takes values between $-E_{\alpha}$ and $E_{\alpha}$. Figure 2 represents the typical shape of the LSR curves for standard and high contact ratio spur gears.

Since the stiffness is defined as the load to deflection ratio and considering eqn. (4), the deflection of the tooth pair in the direction of the line of action is given by [1, 3]:

$$
\delta(\xi)=\frac{F(\xi)}{K_{M}(\xi)}=\frac{F_{T}}{\sum_{j} K_{M}(\xi+j)}
$$

It can be observed that $\delta(\xi)=\delta(\xi+j)$, which means the deflection is equal for all the teeth in simultaneous contact. Consequently, the teeth deflection is described by a relative rotation between both gears, in which the driven gear is delayed respect to the driven one. This conclusion is very interesting and will allow to study the contact at points of modified profile.

However, although $\delta(\xi)$ is equal for all the teeth in contact, it is different at any point of the path of contact as it depends on $\xi$. This means that the delay angle of the driven gear is not uniform, and this variation causes the QSTE to arise. Since the QSTE is defined as the difference between ideal and actual output gear position, it is described by the angle $\varphi_{2}$, as represented in Fig. 3:

$$
\varphi_{2}(\xi)=\frac{\delta(\xi)}{r_{b 2}}=\frac{F_{T}}{r_{b 2}}\left(\sum_{j} K_{M}(\xi+j)\right)^{-1}
$$

Figure 3 represents the instant in which the driving tooth hits the driven tooth at point $I$, outside the line of action and before reaching the theoretical inner point of contact $e$. Point $b$ is described by the intersection of the driving involute profile and the line of action. It is clear that the additional interval of contact due to teeth deflections at the start of contact corresponds to interval

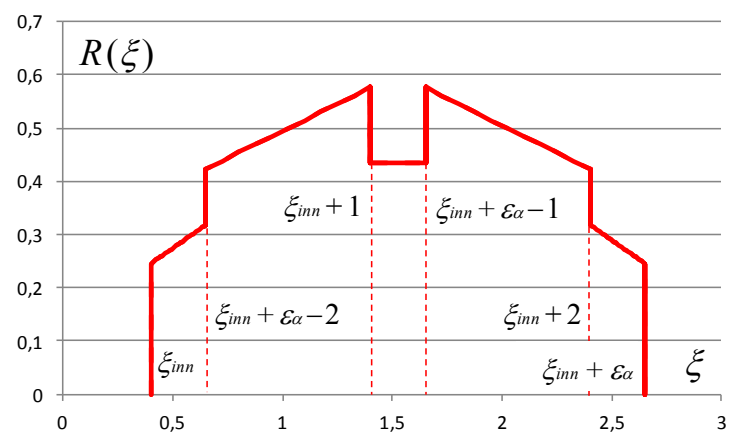

(b) High contact ratio $\left(\varepsilon_{\alpha}=2.25\right)$.

Fig. 2. Load sharing ratio. 


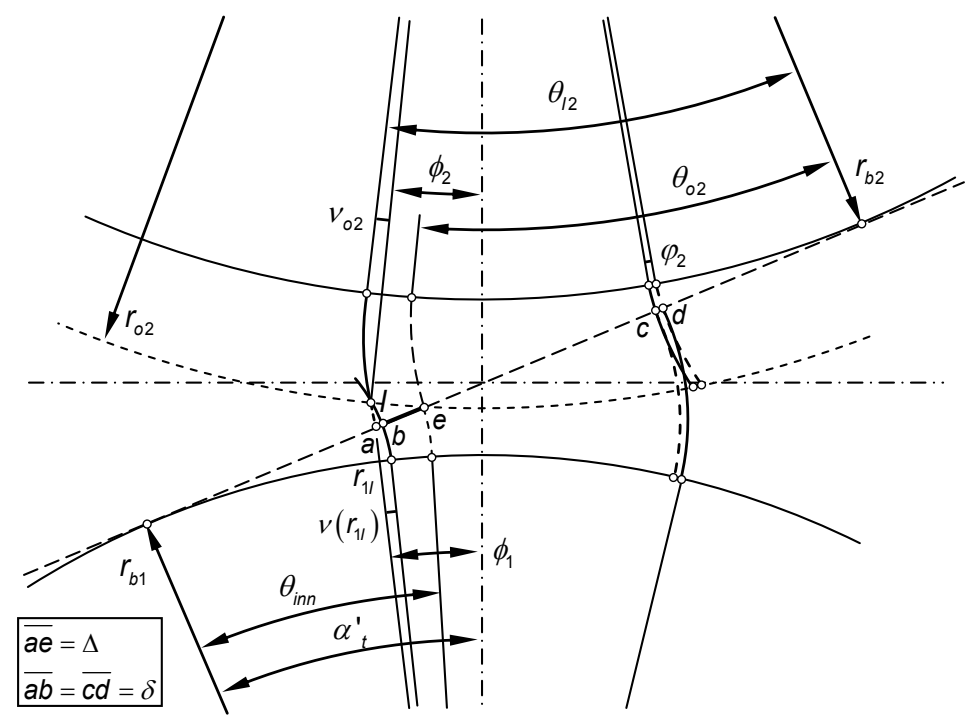

Fig. 3. Actual start of contact of loaded teeth.

$b-e$. A similar additional interval $b^{\prime}-e^{\prime}$, not represented in Fig. 3, arises at the end of contact.

Point $b$ is located below point $e$ at the driving tooth profile, and consequently $\xi_{b}<\xi_{e}$, where $\xi_{e}=\xi_{i n n}$. However, as distance $b-e$ is quite small, it can be assumed $\delta\left(\xi_{b}\right) \approx \delta\left(\xi_{\text {inn }}\right)$, and accounting that the distance $a-b$ is equal to the distance $c-d$ as demonstrated above, $\delta\left(\xi_{\text {inn }}\right)$ can be computed from eqn. (5) just before the contact at point $I$, so that:

$$
\delta \approx \delta\left(\xi_{\text {inn }}\right)=\delta\left(\xi_{\text {inn }}+1\right)=\frac{F_{T}}{\sum_{j>0} K_{M}\left(\xi_{\text {inn }}+j\right)}
$$

Note that the sum is extended from $j=1$ to $E_{\alpha}$, describing the contact conditions just before the hit at point $I$.

If $\Delta$ denotes the distance $a-e$, the lengthening of the contact interval at the start of contact, corresponding to distance $b-e$, is described by $(\Delta-\delta)$ which can be computed from $[1,3]$ :

$$
\left(\frac{\Delta-\delta}{r_{b 1}}\right) \approx \sqrt{\frac{1}{C_{p}}\left(\frac{\delta}{r_{b 1}}\right)}
$$

Similarly, the contact interval lengthening at the end of contact corresponds to $\Delta^{\prime}$ (and not to $\Delta^{\prime}-\delta^{\prime}$ because the rotation is referred to the driving gear), which is equal to distance $b^{\prime}-e^{\prime}$ and can be computed from [3]:

$$
\left(\frac{\Delta^{\prime}}{r_{b 1}}\right) \approx \sqrt{\frac{1}{C_{p}^{\prime}}\left(\frac{\delta^{\prime}}{r_{b 1}}\right)}
$$

where $\delta$ ' corresponds to the teeth deflection just after the end of contact, and therefore:

$$
\delta^{\prime} \approx \delta\left(\xi_{i n n}+d_{\alpha}\right)=\frac{F_{T}}{\sum_{j<E_{\alpha}} K_{M}\left(\xi_{i n n}+d_{\alpha}+j\right)}
$$

Here, the sum limit $j<E_{\alpha}$ means from $j=0$ to $E_{\alpha}-1$.
The calculation of coefficients $C_{p}$ and $C_{p}^{\prime}$ can be found in [3].

The last step of the analysis of the contact between unmodified teeth is the determination of the LSR along the additional contact intervals. If $\phi$ and $\phi^{k}$ are the angles rotated by the driving gear along the inner and outer additional contact intervals, respectively, these intervals are described by:

$$
\begin{gathered}
0 \leq \phi \leq \frac{(\Delta-\delta)}{r_{b 1}}=\phi_{\text {max }} \\
0 \leq \phi^{\prime} \leq \frac{\Delta^{\prime}}{r_{b 1}}=\phi_{\text {max }}^{\prime}
\end{gathered}
$$

and taking into account that $\phi$ and $\phi$ are related with $\xi$ by:

$$
\begin{gathered}
\xi=\xi_{i n n}-\frac{z_{1}}{2 \pi}\left(\phi_{\max }-\phi\right) \\
\xi=\xi_{i n n}+\varepsilon_{\alpha}+\frac{z_{1}}{2 \pi} \phi^{\prime}
\end{gathered}
$$

the additional contact intervals can be also expressed in terms of $\xi$ as:

$$
\begin{array}{ll}
\xi_{\text {min }} \leq \xi \leq \xi_{\text {inn }} & \left(\xi_{\text {min }}=\xi_{\text {inn }}-\frac{z_{1}}{2 \pi} \phi_{\max }\right) \\
\xi_{\text {inn }}+\varepsilon_{\alpha} \leq \xi \leq \xi_{\max } & \left(\xi_{\text {max }}=\xi_{\text {inn }}+\varepsilon_{\alpha}+\frac{z_{1}}{2 \pi} \phi_{\text {max }}^{\prime}\right)
\end{array}
$$

The meshing stiffness along the additional contact intervals can be computed by following a similar procedure as one described in [7]. Result is shown in Fig. 4. It can be observed that its values are almost uniform along both additional contact intervals, and therefore they can be all considered approximately equal to the values at both limits of the theoretical contact interval.

According to this, the equation of the meshing stiffness along the extended contact interval will be: 


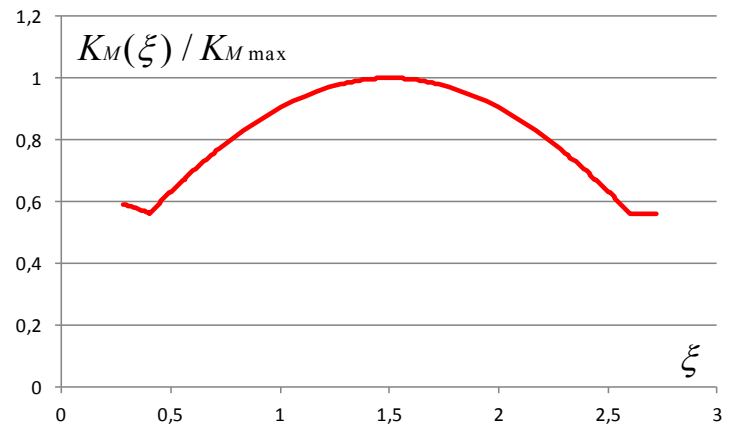

Fig. 4. Extended meshing stiffness $\left(\varepsilon_{\alpha}=2.20\right)$.

$$
\begin{array}{ll}
K_{M}(\xi)=K_{M \max } \cos \left(b_{0} \frac{\varepsilon_{\alpha}}{2}\right) & \xi_{\text {min }} \leq \xi \leq \xi_{\text {inn }} \\
K_{M}(\xi)=K_{M \max } \cos \left(b_{0}\left(\xi-\xi_{m}\right)\right) & \xi_{\text {inn }} \leq \xi \leq \xi_{\text {inn }}+\varepsilon_{\alpha} \\
K_{M}(\xi)=K_{M \max } \cos \left(b_{0} \frac{\varepsilon_{\alpha}}{2}\right) & \xi_{\text {inn }}+\varepsilon_{\alpha} \leq \xi \leq \xi_{\max }
\end{array}
$$

The rotation angle inside the inner additional contact interval verifies:

$$
(\Delta-\delta)_{\phi=0}-(\Delta-\delta)_{\phi}=r_{b 1} \phi
$$

From Fig. 3, $(\Delta-\delta)_{\phi=0}$ is equal to $r_{b} \phi_{\max }$, which can be computed with eqns. (7) and (8). Consequently:

$$
(\Delta-\delta)_{\phi}=(\Delta-\delta)_{\phi=0}-r_{b 1} \phi=r_{b 1}\left(\phi_{\max }-\phi\right)
$$

and, from eqn. (8):

$$
(\delta)_{\phi}=C_{p} r_{b 1}\left(\frac{(\Delta-\delta)_{\phi}}{r_{b 1}}\right)^{2}=C_{p} r_{b 1}\left(\phi_{\max }-\phi\right)^{2}
$$

$(\delta)_{\phi}$ represents the required deflection of the previous tooth pairs to ensure the start of contact at point described by $\phi$. Henceforward it will be represented by $\delta_{G}(\phi)$ to clarify the notation. Accordingly, for contact at point $\phi$, if the deflection of the previous tooth pairs in contact $(j>0)$ is $\delta(\phi)$, the deflection of the new pair in contact $(j=0)$ will be $\delta(\phi)-\delta_{G}(\phi)$. Consequently, the load at each pair will be given by:

$$
\begin{gathered}
F_{0}(\phi)=K_{M 0}(\phi)\left(\delta(\phi)-\delta_{G}(\phi)\right) \\
F_{j>0}(\phi)=K_{M j}(\phi) \delta(\phi)
\end{gathered}
$$

In eqn. (18), $K_{M j}(\phi)$ represents the meshing stiffness of tooth pair $j$ when tooth pair 0 contacts at the position described by $\phi$ (inside the interval $0 \leq \phi \leq \phi_{\max }$ ). Obviously, $K_{M j}(\xi)=K_{M}(\xi+j)$, and therefore $K_{M j}(\phi)=$ $K_{M}\left(\phi+j 2 \pi / z_{1}\right)$. Then, according to eqn. (14), eqn. (18) may be written as:

$$
\begin{gathered}
F_{0}(\xi) \approx K_{M}\left(\xi_{i n n}\right)\left(\delta(\phi)-\delta_{G}(\phi)\right) \\
F_{j>0}(\xi)=K_{M}(\xi+j) \delta(\phi)
\end{gathered}
$$

where $\phi$ and $\xi$ are related by eqn. (12).

From eqn. (19), the total load is:

$$
\begin{aligned}
F_{T} & =\sum_{j \geq 0} F_{j}(\phi) \\
& =\sum_{j \geq 0} K_{M}(\xi+j) \delta(\phi)-K_{M}\left(\xi_{i n n}\right) \delta_{G}(\phi)
\end{aligned}
$$

and consequently the tooth deflection is given by:

$$
\delta(\phi)=\frac{F_{T}+K_{M}\left(\xi_{\text {inn }}\right) \delta_{G}(\phi)}{\sum_{j \geq 0} K_{M}(\xi+j)}
$$

Since the total load does not change along the path of contact, $F_{T}$ can be computed from eqn. (20) just before the start of contact, for $\phi=0$ and $\xi=\xi_{\text {min }}$, and therefore, accounting eqn. (17):

$$
\begin{aligned}
F_{T} & =\sum_{j>0} K_{M}\left(\xi_{\min }+j\right)(\delta)_{\phi=0} \\
& =\sum_{j>0} K_{M}\left(\xi_{\min }+j\right) C_{p} r_{b 1} \phi_{\max }^{2}
\end{aligned}
$$

Finally, replacing eqns. (17) and (22) in eqn. (21), and the result in eqn. (19), the LSR along the additional inner contact interval is given by:

$$
\begin{array}{r}
R_{0}(\xi)=\frac{K_{M}\left(\xi_{\text {inn }}\right)}{\sum_{j \geq 0} K_{M}(\xi+j)}\left(1-\frac{\sum_{j>0} K_{M}(\xi+j)}{\sum_{j>0} K_{M}\left(\xi_{\min }+j\right)}\left(1-\frac{\phi}{\phi_{\max }}\right)^{2}\right) \\
R_{i>0}(\xi)=\frac{K_{M}(\xi+i)}{\sum_{j \geq 0} K_{M}(\xi+j)}\left(1+\frac{K_{M}\left(\xi_{\text {inn }}\right)}{\sum_{j>0} K_{M}\left(\xi_{\min }+j\right)}\left(1-\frac{\phi}{\phi_{\max }}\right)^{2}\right)
\end{array}
$$

which is valid for $\xi_{\min } \leq \xi_{\leq} \xi_{\text {inn }}$. Following an identical procedure with the outer contact interval, accounting that the rotation angle verifies:

$$
\left(\Delta^{\prime}\right)_{\phi^{\prime}}-\left(\Delta^{\prime}\right)_{\phi^{\prime}=0}=\left(\Delta^{\prime}\right)_{\phi^{\prime}}=r_{b 1} \phi^{\prime}
$$

and from eqns. (24) and (9):

$$
\left(\delta^{\prime}\right)_{\phi^{\prime}}=C_{p}{ }_{p} r_{b 1} \phi^{\prime 2}
$$

it is obtained:

$R_{E_{\alpha}}(\xi)=$

$\frac{K_{M}\left(\xi_{\text {inn }}+\varepsilon_{\alpha}\right)}{\sum_{j \leq E_{\alpha}} K_{M}\left(\xi-E_{\alpha}+j\right)}\left(1-\frac{\sum_{j<E_{\alpha}} K_{M}\left(\xi-E_{\alpha}+j\right)}{\sum_{j<E_{\alpha}} K_{M}\left(\xi_{\max }-E_{\alpha}+j\right)}\left(\frac{\phi^{\prime}}{\phi_{\text {max }}^{\prime}}\right)^{2}\right)$

$R_{i<E_{\alpha}}(\xi)=$

$\frac{K_{M}\left(\xi-E_{\alpha}+i\right)}{\sum_{j \leq E_{\alpha}} K_{M}\left(\xi-E_{\alpha}+j\right)}\left(1+\frac{K_{M}\left(\xi_{\text {inn }}+\varepsilon_{\alpha}\right)}{\sum_{j<E_{\alpha}} K_{M}\left(\xi_{\max }-E_{\alpha}+j\right)}\left(\frac{\phi^{\prime}}{\phi_{\text {max }}^{\prime}}\right)^{2}\right)$ 


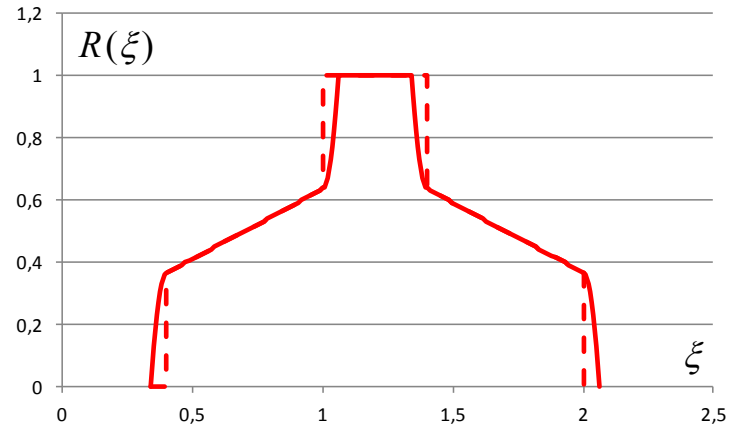

(a) Standard contact ratio $\left(\varepsilon_{\alpha}=1.60\right)$.

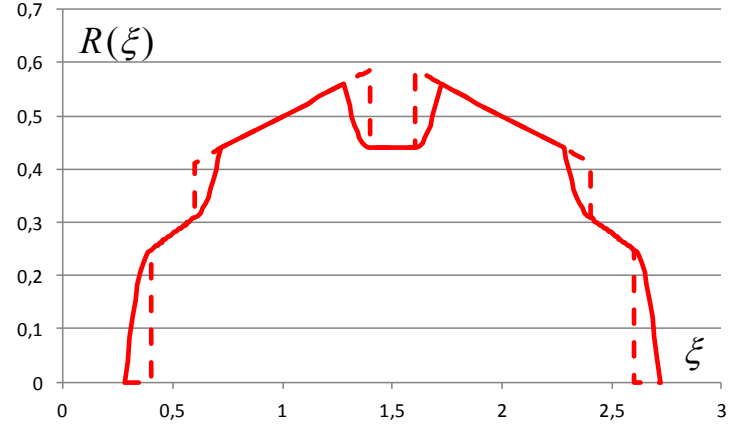

(b) High contact ratio $\left(\varepsilon_{\alpha}=2.20\right)$.

Fig. 5. Curves of load sharing ratio along the extended contact interval.

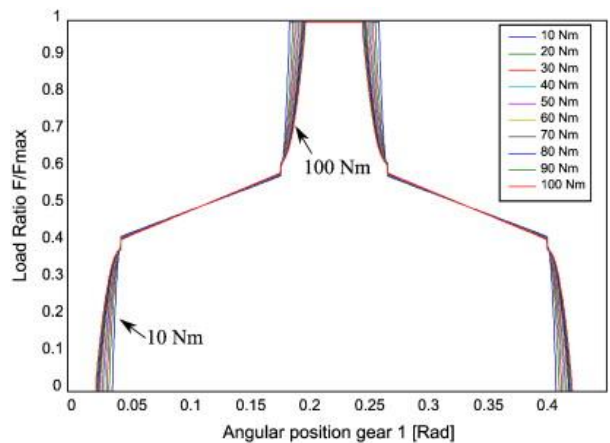

Fig. 6. LSR obtained from FE analysis [2].

Figure 5 represents the LSR along the extended interval of contact, obtained from eqns. (23) and (26), for standard and high contact ratio spur gears. Fig 6 reproduces the diagram presented by Fernández del Rincón et al. in [2], obtained by FEM analyses. It is remarkable the similarity between this diagram in Fig. 5(a) and one in Fig. 6.

Finally, replacing eqns. (22) and (17) in eqn. (21):

$$
\begin{aligned}
& \delta(\phi)= \\
& C_{p} r_{b 1} \phi_{\max }^{2} \frac{\sum_{j>0} K_{M}\left(\xi_{\min }+j\right)}{\sum_{j \geq 0} K_{M}(\xi+j)}\left(1+\frac{K_{M}\left(\xi_{\text {inn }}\right)}{\sum_{j>0} K_{M}\left(\xi_{\min }+j\right)}\left(1-\frac{\phi}{\phi_{\max }}\right)^{2}\right)
\end{aligned}
$$

from which the QSTE is given by:

$$
\varphi_{2}(\phi)=\frac{\delta(\phi)}{r_{b 2}}
$$

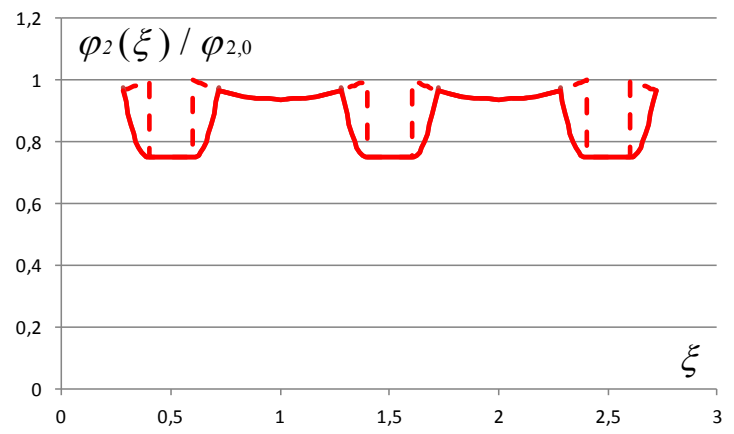

Fig. 7. QSTE for unmodified teeth $\left(\varepsilon_{\alpha}=2.20\right)$.
Figure 7 represents the curve of QSTE for high contact ratio spur gear.

\section{Tooth modifications}

Profile modifications can be used for avoiding the shock between teeth at the start of contact. From Fig. 3, if the driven tooth were relieved an amount $\delta_{R}=(\delta)_{\phi=0}$ at the tip, the start of contact will be delayed to the theoretical inner point of contact and the mesh will begin smoothly, at the proper place and without shocks.

Figure 8 shows the geometry of a tip-relieved involute tooth. $\delta_{R}$ is the amount of modification, which is measured perpendicularly to the involute profile and should be equal to the teeth deflection at the inner point of contact, just before the contact of the new tooth, i.e. $\delta_{R}=(\delta)_{\phi=0}=\delta\left(\xi_{i n n}\right)$. The interval of modification is defined by the inner limit $\xi_{r l}$ and the tooth tip point $\xi_{o}=$ $\xi_{\text {inn }}+\varepsilon_{\alpha}$. The length of modification is the distance between both limits, which will be expressed in terms of $\xi$, as $\Delta \xi_{r}=\xi_{o}-\xi_{r l}$. The shape of modification is the shape of the profile inside the interval of profile modification, and will be expressed as $\delta_{R}=\delta_{R}(\xi)$, always measured perpendicularly to the involute profile at point $\xi$. However, in this work, the profile modification will be expressed as a function of the contact point on the line of action. Accordingly, a tip relief at the driven tooth will be described by $\delta_{R}(\xi)$, for $\xi_{\text {inn }} \leq \xi \leq \xi_{\text {inn }}+\Delta \xi_{\text {r }}$, where $\delta_{R}\left(\xi_{\text {inn }}+\Delta \xi_{r}\right)=0$. A tip relief at the driving tooth will be described by $\delta^{\prime}{ }_{R}(\xi)$, for $\xi_{\text {inn }}+\varepsilon_{\alpha}-\Delta^{\prime} \xi_{r} \leq \xi \leq \xi_{\text {inn }}+\varepsilon_{\alpha}$ where $\delta_{R}^{\prime}\left(\xi_{\text {inn }}+\varepsilon_{\alpha}-\Delta^{\prime} \xi_{r}\right)=0$.

The deflection of a couple of teeth $j$ at contact point $\xi$ will be the difference between the gap due to the delay

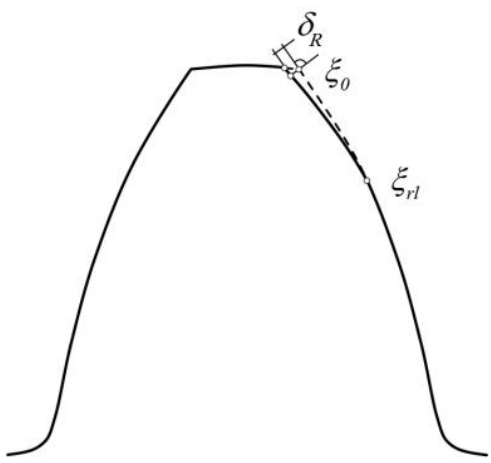

Fig. 8. Tip relieved spur tooth. 


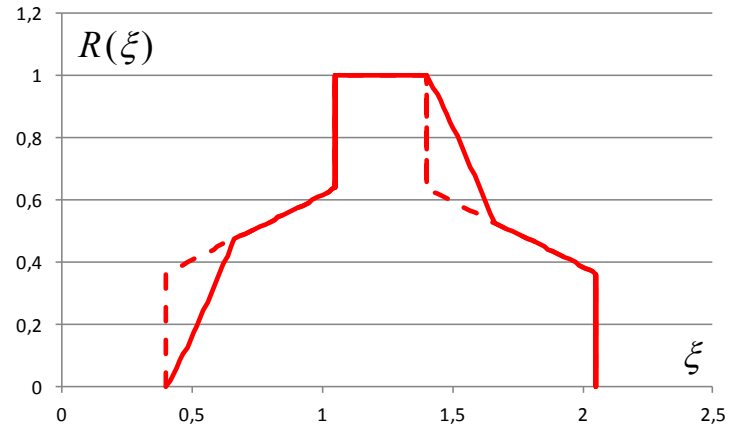

(a) Theoretical LSR.

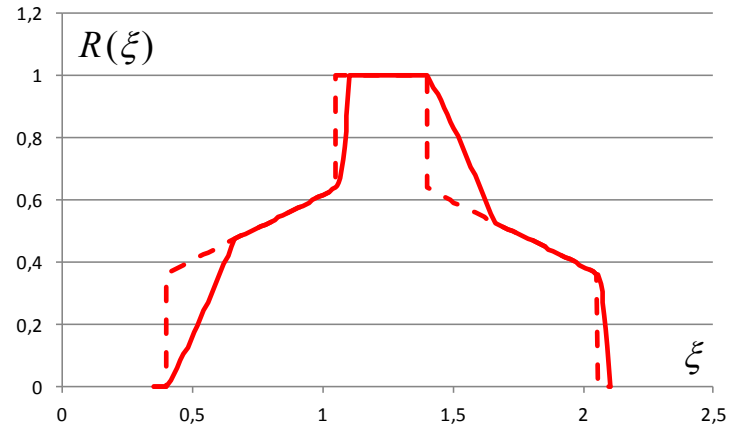

(b) Actual LSR. angle and the amount of modification at this point:

$$
\delta_{j}(\xi)=\delta(\xi)-\delta_{R j}(\xi)
$$

Accordingly, the load at any pair:

$$
F_{j \geq 0}(\xi)=K_{M j}(\xi)\left(\delta(\xi)-\delta_{R j}(\xi)\right)
$$

where $K_{M j}(\xi)=K_{M}(\xi+j)$ and $\delta_{R j}(\xi)=\delta_{R}(\xi+j)$. From eqn. (30):

$$
\begin{gathered}
F_{T}=\sum_{j \geq 0} K_{M j}(\xi) \delta(\xi)-\sum_{j \geq 0} K_{M j}(\xi) \delta_{R j}(\xi) \\
\delta(\xi)=\frac{F_{T}+\sum_{j \geq 0} K_{M j}(\xi) \delta_{R j}(\xi)}{\sum_{j \geq 0} K_{M j}(\xi)}
\end{gathered}
$$

and, replacing eqn. (31) in eqn. (30):

$$
R_{i}(\xi)=\frac{K_{M i}(\xi)}{\sum_{j \geq 0} K_{M j}(\xi)}\left(1+\frac{1}{F_{T}} \sum_{j \geq 0} K_{M j}(\xi)\left(\delta_{R j}(\xi)-\delta_{R i}(\xi)\right)\right)
$$

where $\delta_{R j}(\xi)=0$ at least for one $j$ to ensure the continuity of the transmission. For standard contact ratio spur gears $\left(1<\varepsilon_{\alpha}<2\right)$ this condition implies the length of modification to be smaller than the fractional part of $\varepsilon_{\alpha}$, because the profile cannot be modified along the interval of one pair tooth contact. For this condition $\Delta \xi_{r}<d_{\alpha}$, it is verified $\delta_{R j}(\xi)=0$ for $j \neq 0$, and the total load can be computed just after the start of contact at $\xi_{\text {inn }}$ as:

$$
F_{T}=\sum_{j>0} K_{M j}\left(\xi_{i n n}\right) \cdot \delta\left(\xi_{i n n}\right)
$$

Taking this into account, eqn. (32) results in:

$$
\begin{gathered}
R_{0}(\xi)=\frac{K_{M 0}(\xi)}{\sum_{j \geq 0} K_{M j}(\xi)}\left(1-\frac{\sum_{j>0} K_{M j}(\xi) \delta_{R 0}(\xi)}{\sum_{j>0} K_{M j}\left(\xi_{i n n}\right) \cdot \delta\left(\xi_{i n n}\right)}\right) \\
R_{i>0}(\xi)=\frac{K_{M i}(\xi)}{\sum_{j \geq 0} K_{M j}(\xi)}\left(1+\frac{K_{M 0}(\xi) \delta_{R 0}(\xi)}{\sum_{j>0} K_{M j}\left(\xi_{i n n}\right) \cdot \delta\left(\xi_{i n n}\right)}\right)
\end{gathered}
$$

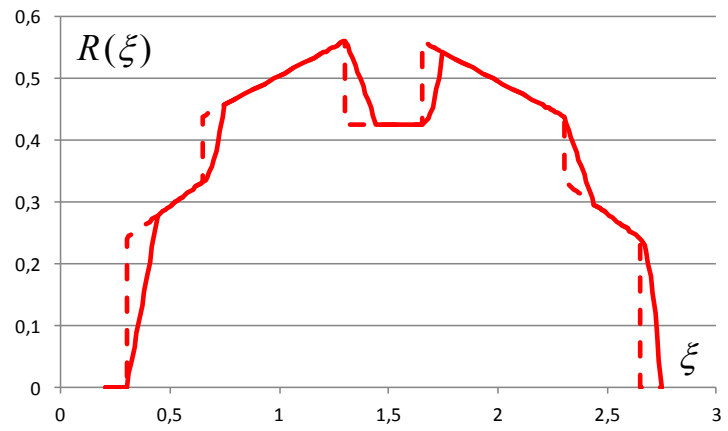

Fig. 10. Actual LSR for linear tip relief and high contact ratio $\left(\varepsilon_{\alpha}=2.35, \Delta \xi_{r}=0.14\right)$.

The curve of LSR described by eqn. (34) for linear tip relief has been represented in Fig. 9(a). However, this is only a theoretical result, because the tip relief shifts the start of contact to the theoretical inner point of contact $\xi_{\text {inn }}$, but if relief is not symmetric, the contact interval is extended at the end of contact, as discussed in the previous section.

The actual LSR is represented in Fig. 9(b). The curve was obtained by the superposition of curves described by eqns. (26) and (34); the first one extended along the interval of $\xi$ corresponding to the interval $0 \leq \phi \leq \phi_{\max }$, and the other extended along the rest of the contact interval. Figure 10 represents the actual LSR for high contact ratio spur gears $\left(2 \leq \varepsilon_{\alpha}<3\right)$.

Actual curves of LSR represented in Figs. 9(b) and 10 are valid thanks to the limitation on the length of relief $\Delta \xi_{r}<d_{\alpha}$ is regarded. For these short relieves, simultaneous contact at the relieved interval and at the extended interval, in different contacting pairs, is not possible. In fact, relieved contact interval is contained in the maximum tooth pair contact interval while the extended interval due to deflections affects the theoretical interval of minimum tooth pair contact. And consequently, equation (34) is valid and superposition of equations (34) and (26) is valid as well.

For $d_{\alpha}<\Delta \xi_{r}<1$ (named henceforward as long tip relief), eqn. (34) remains to be valid, but the superposition of equations (26) and (34) is no longer valid. For $\Delta \xi_{r}>1$.

Figure 11 represents the LSR for a high contact ratio spur pair with long tip relief, obtained from eqn. (34) but regardless the additional contact interval at the end of contact. Consequently, the diagram in Fig. 11 represents 


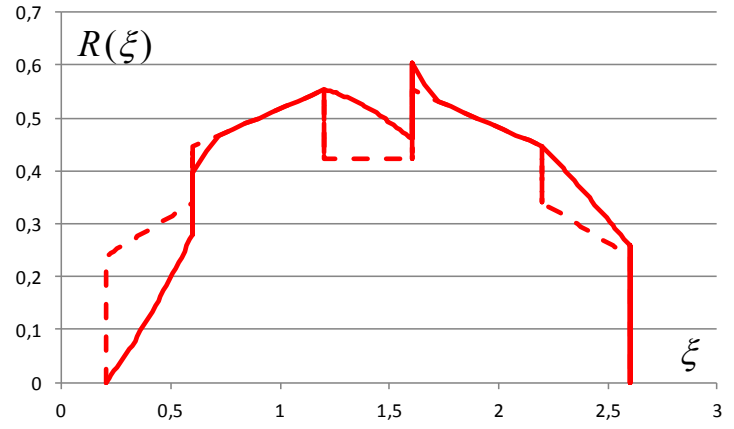

Fig. 11. Theoretical LSR for high contact ratio and long tip relief $\left(\varepsilon_{\alpha}=2.4, \Delta \xi_{r}=0.50\right)$.

only a theoretical curve of LSR, perhaps valid for weakly loaded teeth.

This result shows the effect of long tip relief on the teeth load very clearly: the load decreases at the lower part of the contact interval, and increases at the upper part. This means a better behaviour against pitting and wear (as the critical contact stress is always located at the inner contact points), and a little worse behaviour against bending fatigue (as the critical tooth root stress is always located at the outer contact points). However, two aspects should be accounted for the discussion:

1. The behaviour against pitting is usually much more critical than the behaviour against bending.

2. The parabolic downloading of the upper tooth pair suggests that the peak of the load at the inner point of the outer interval of two pair tooth contact could be absorbed. Consequently, the maximum load along the whole contact interval will not increase.

In conclusion, long tip relieves could be used for balanced pitting/bending strength designs, with higher critical safety margins. This justifies the development of a model of load transfer for long tip relief, which is presented in the next section.

\section{Long tip relief}

For a spur gear with tip relieved profile on the driven gear and unmodified profile on the driving gear, according to the notation above, the effective interval of contact is described by:

$$
\xi_{\text {inn }} \leq \xi \leq \xi_{\max }
$$

In a general way, the deflection of a couple of teeth

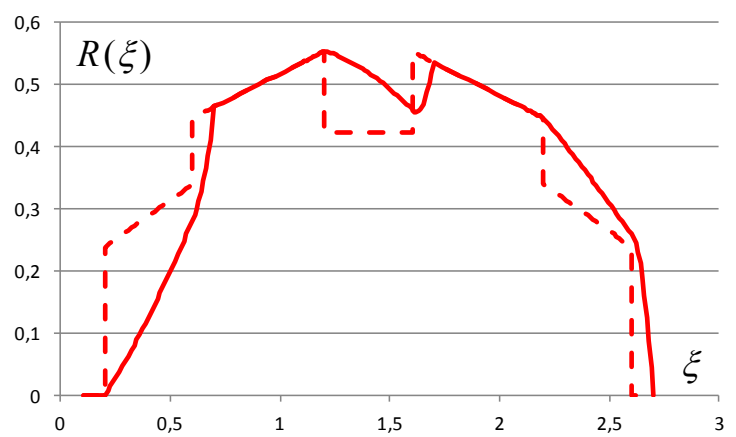

(a) Adjusted length of relief $\left(\Delta \xi_{r}=0.50\right)$. can be expressed as:

$$
\delta_{j}(\xi)=\delta(\xi)-\left(\delta_{R j}(\xi)+\delta_{G j}(\xi)\right)
$$

where:

$\begin{array}{ll}\delta_{R j}(\xi)=0 & \text { for } j>0 \text { or } \xi>\xi_{\text {inn }}+\Delta \xi_{r} \\ \delta_{G j}(\xi)=0 & \text { for } j<E_{\alpha} \text { or } \xi<\xi_{\text {inn }}+d_{\alpha} \text { or } \xi>\xi_{\max }-E_{\alpha}\end{array}$

Following an identical procedure as one presented in Section 2, eqns. (29) to (32), the load at any tooth pair is given by:

$$
F_{j}(\xi)=K_{M j}(\xi)\left(\delta(\xi)-\left(\delta_{R j}(\xi)+\delta_{G j}(\xi)\right)\right)
$$

and therefore:

$$
\begin{gathered}
F_{T}=\sum_{j \geq 0} K_{M j}(\xi) \delta(\xi)-\sum_{j \geq 0} K_{M j}(\xi)\left(\delta_{R j}(\xi)+\delta_{G j}(\xi)\right) \\
\delta(\xi)=\frac{F_{T}+\sum_{j \geq 0} K_{M j}(\xi)\left(\delta_{R j}(\xi)+\delta_{G j}(\xi)\right)}{\sum_{j \geq 0} K_{M j}(\xi)}
\end{gathered}
$$

and, replacing eqn. (39) in eqn. (38), the LSR is given by:

$R_{i}(\xi)=$

$\frac{K_{M i}(\xi)}{\sum_{j \geq 0} K_{M j}(\xi)}\left(1+\frac{\sum_{j \geq 0} K_{M j}(\xi)\left(\left(\delta_{R j}(\xi)+\delta_{G j}(\xi)\right)-\left(\delta_{R i}(\xi)+\delta_{G i}(\xi)\right)\right)}{F_{T}}\right)$

Figure 12 shows the actual LSR for a high contact ratio spur gear with long tip relief, obtained from eqn. (40). In fact, the parabolic downloading of the upper tooth pair induces a parabolic loading process of the next pair absorbing the peak of the theoretical curve of LSR. However, if the tip relief is too long the peak is not completely absorbed as the profile modification is extended beyond the interval of parabolic loading, as seen in Fig. 12(b). Hence the length of relief should not be greater than the length of the inner interval of minimum pair tooth contact plus the length of the additional contact interval at the outer limit $\Delta \xi_{o}$, or what is the same:

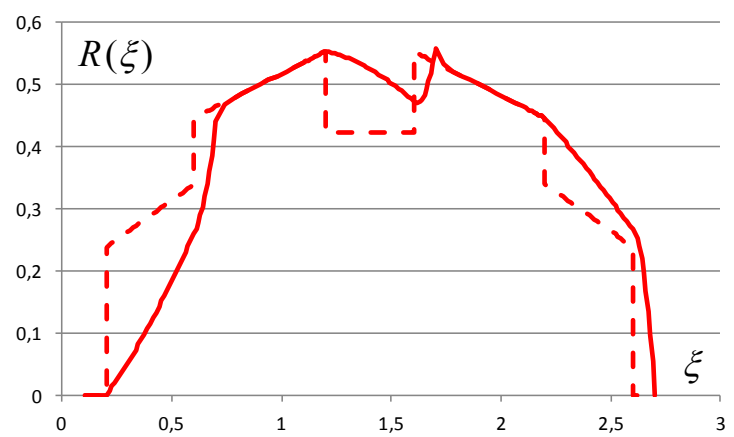

(b) Too long relief $\left(\Delta \xi_{r}=0.55\right)$.

Fig. 12. Actual LSR for high contact ratio spur gears with long tip relief $\left(\varepsilon_{\alpha}=2.4, \Delta \xi_{o}=0.1\right)$. 


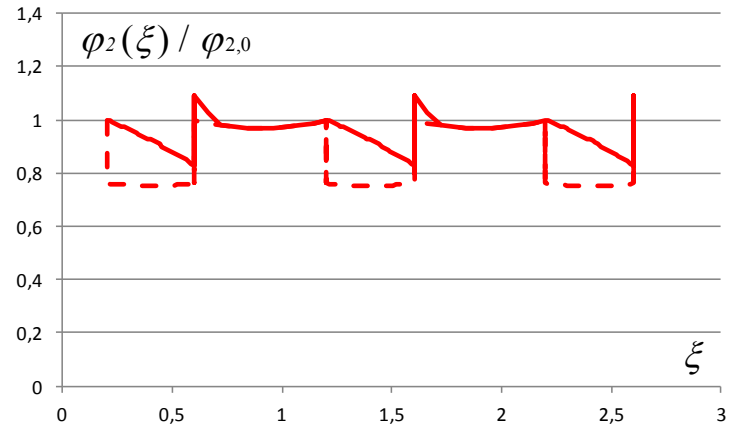

(a) Theoretical QSTE $\left(\Delta \xi_{o}=0, \Delta \xi_{r}=0.50\right)$.

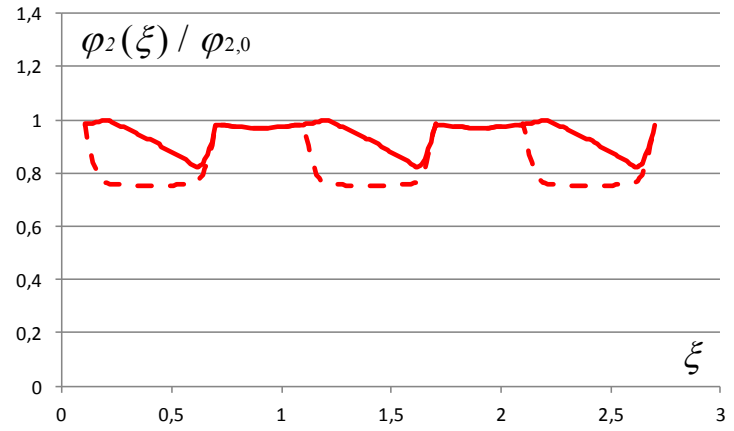

(b) QSTE for adjusted relief $\left(\Delta \xi_{o}=0.1, \Delta \xi_{r}=0.50\right)$.

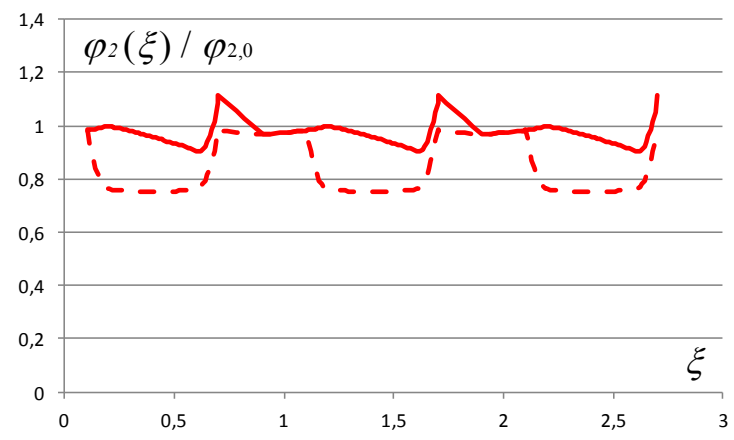

(c) QSTE for too long relief $\left(\Delta \xi_{o}=0.1, \Delta \xi_{r}=0.70\right)$.

Fig. 13. Curves of QSTE for high contact ratio and long tip relief $\left(\varepsilon_{\alpha}=2.4\right)$.

$$
\begin{aligned}
\Delta \xi_{r} & <d_{\alpha}+\Delta \xi_{o}=d_{\alpha}+\left(\xi_{\max }-\left(\xi_{\text {inn }}+\varepsilon_{\alpha}\right)\right) \\
& =\xi_{\max }-\xi_{\text {inn }}-E_{\alpha}
\end{aligned}
$$

Interesting conclusions can be also obtained from the analysis of the QSTE. The expression of $\delta$ in eqn. (39) describes the QSTE since the delay angle of the driven gear is proportional to the teeth deflection, as shown in eqn. (28).

Figure 13(a) shows the theoretical QSTE, obtained from eqn. (39) for $\delta_{G j}(\xi)=0$. It can be observed that the fluctuation of the QSTE (which governs the fluctuation of the output velocity) is more or less the same as that for no tip relief. (The numerical analysis reveals that the fluctuation of QSTE for modified profile is, in this example, $4.5 \%$ higher than that for unmodified profile).

Figure 13(b) shows the actual QSTE, obtained from eqn. (39) for $\delta_{G j}(\xi) \neq 0$, as described by eqn. (25). In this case, the fluctuation of the QSTE is significantly reduced (almost 30\% reduction in the example). This reduction is not possible for short tip relieves, in which both curves of QSTE, for modified and unmodified profiles, are coincident at any point of the interval of maximum tooth pair contact interval, and consequently at the interval of minimum QSTE, as shown in Figs. 13(a) and 13(b). Since both curves of QSTE along the intervals of minimum tooth pair contact should be necessarily coincident, the maximum QSTE will be the same, and therefore the fluctuation of QSTE cannot be reduced.

As shown in Fig. 13(b), the maximum QSTE for modified teeth is equal to one for unmodified teeth, and is reached at both limits of the minimum tooth pair contact intervals. But this is true only if condition for length of modification $\Delta \xi_{r}<d_{\alpha}+\Delta \xi_{o}$ is regarded; on the contrary the peak in Fig. 13(a) will appear and the maximum QSTE will increase, as represented in Fig. 13(c) for too long tip relief.

On the other hand, it is clear that as the length of relief is reduced the QSTE curve of modified profile intersects the QSTE curve of unmodified profile at lower points, until reaching the intersection at minimum QSTE interval for $\Delta \xi_{r}<d_{\alpha}$, for which the fluctuation of QSTE is maximum. In conclusion, the shorter the length of tip relief the greater the fluctuation of QSTE. Accordingly, the optimum length of relief will be:

$$
\left(\Delta \xi_{r}\right)_{\mathrm{opt}}=d_{\alpha}+\Delta \xi_{o}
$$

for which the fluctuation of QSTE is minimum and the pitting and bending strengths can be balanced.

\section{Conclusions}

In this work, a study on the load sharing and the transmission error for high contact ratio spur gears with long profile modification has been performed, based on models of meshing stiffness and tooth deflections previously developed.

Long tip relieves, beyond the inner interval of two pair tooth contact provides smaller values of the critical contact stress (usually located at the inner part of the contact interval), keeping the values of the critical tooth root stress unaltered, or slightly increased. This means that these long tip relieves can be used for balanced pitting/bending strength spur gears.

The fluctuation of the QSTE can be also reduced by the appropriate choice of the length of tip relief. It has been observed that the shorter the length of tip relief the 
higher the minimum QSTE. However, the maximum QSTE also increases as the curve presents a peak at the inner limit of the interval of minimum tooth pair contact. But this peak does not appear if the interval of profile modification does not exceed the additional contact interval induced by teeth deflections, because inside this interval the peak is absorbed by the parabolic downloading of the upper tooth pair. Consequently, for this adjusted length of relief the maximum QSTE keeps its value, while the minimum one increases, from which the fluctuation of the QSTE is reduced.

From all these considerations, it can be concluded that the optimum length of relief is equal to the length of the maximum tooth pair contact interval (i.e. the fractional part of the contact ratio) plus the length of the additional contact interval due to teeth deflection.

Thanks are expressed to the Spanish Council for Scientific and Technological Research for the support of the project DPI2015-69201-C2-1-R, "Load Distribution and Strength Calculation of Gears with Modified Geometry", as well as to the School of Engineering of UNED for the support of the action 2017-MEC27, "Calculation Models of Cylindrical Gears".

\section{References}

1. J.I. Pedrero, M. Pleguezuelos, M.B. Sánchez, Proc. International Conference on Gears, 69 (2017)

2. A. Fernández del Rincón et al., Mech. Mach. Theory 61, 30-58 (2013)

3. J.I. Pedrero, M. Pleguezuelos, M.B. Sánchez, Revista Iberoamericana de Ingeniería Mecánica 22, 71-90 (2018)

4. H.H. Lin, F.B. Oswald, D.P. Townsend, Mech. Mach. Theory 29, 1115-1129 (1994)

5. J. Wang, I. Howard, Proc. International Conference on Mechanical Transmissions, 42-47 (2006)

6. Y.A. Tesfahunegn, F. Rosa, C. Gorla, Proc. Inst. Mech. Eng. Pt. C J. Mechan. Eng. Sci. 224, 17491758 (2010)

7. M.B. Sánchez, M. Pleguezuelos, J.I. Pedrero, Mech. Mach. Theory 109, 231-249 (2017) 